\title{
Fusing Deep Learning and Sparse Coding for SAR ATR
}

\author{
Odysseas Kechagias-Stamatis and Nabil Aouf
}

\begin{abstract}
We propose a multi-modal and multi-discipline data fusion strategy appropriate for Automatic Target Recognition (ATR) on Synthetic Aperture Radar imagery. Our architecture fuses a proposed Clustered version of the AlexNet Convolutional Neural Network with Sparse Coding theory that is extended to facilitate an adaptive elastic net optimization concept. Evaluation on the MSTAR dataset yields the highest ATR performance reported yet which is $99.33 \%$ and $99.86 \%$ for the 3 and 10 -class problems respectively.
\end{abstract}

Index Terms-Automatic Target Recognition, Convolutional Neural Networks, Data Fusion, Sparse Coding, Synthetic Aperture Radar

\section{INTRODUCTION}

$\mathbf{M}^{\circ}$ ODERN warfare requires high performing Automatic Target Recognition (ATR) algorithms to avoid collateral damage and fratricide. During the last decades, both industry and academia have made several ATR attempts in various data domains such as 2D Infrared [1], 3D Light Detection and Ranging (LIDAR) [2]-[4] and 2D Synthetic Aperture Radar [5]-[19] (SAR). Despite each data modality having its own advantages, SAR imagery is appealing because it can be obtained under all-weather night-and-day conditions extending considerably the operational capabilities in the battlefield. Due to these advantages, SAR ATR has been attempted using various techniques.

Suggested methods include feature-based solutions where the SAR image is described by a set of robust attributes capable of achieving target classification under various nuisance factors. Feature-based solutions may rely on Krawtchouk moments [20] where features are derived from the discrete-defined Krawtchouk polynomials or on biologically inspired features. The latter can rely on episodic and semantic features [21] or sparse robust filters [22] that originate from the human cognition process. Other methods include binary operations [23], using the target's scattering centers [15], [24] or the azimuth and range target profiles fusion [25].

Another type of SAR ATR algorithms uses a Stacked Autoencoder (SA) that extracts features from SAR imagery and inputs them to an SA type neural network. The latter is an

O. Kechagias-Stamatis and N. Aouf are with the Signals and Autonomy Group, Centre for Electronic Warfare Information and Cyber, Cranfield University, Defense Academy of the UK, Shrivenham, SN6 8LA, UK (e-mail: o.kechagiasstamatis@cranfield.ac.uk) unsupervised learning structure used in neural networks that can convert the input data into abstract expressions utilizing a non-linear model. SA type SAR ATR suggests either exploiting Local Binary Features [18] or modifying the reconstruction error of the typical autoencoder scheme by adding an Euclidean distance restriction for the hidden layer features [17]. Other autoencoder based solutions are influenced by the human visual cortical system [26] or are combined with a Synergetic neural network concept [27].

Compressive Sensing (CS) has also been used for SAR ATR to recover the SAR signal that has been remapped from the originating domain into a domain where the signal is sparse using a non-adaptive linear projection. Signal recovery is achieved via an $l_{l}$-norm optimization process. For example, Multitask CS [28] exploits the statistical correlation among multiple target views to recover the target's signature that is then used for target recognition under a compressive sensing scheme. Bayesian CS [14] relies on the scattering centers of the SAR image that are used as an input signal to the CS technique.

Sparse Representation Classification (SRC) or Sparse Coding (SC) type of solutions aim at recovering the SAR testing imagery out of a dictionary where the SAR training images are the dictionary's base elements. SRC aims at identifying the sparsest representation of the testing imagery within the dictionary by employing an $l_{1}$-norm optimization scheme. The final classification decision mechanism matches the class that provides the smallest residual error. Joint SRC [29] for example, exploits three target views to increase the completeness of the target's SAR signature and a mixed $l_{0} \mid l_{2}-$ norm. The reasoning of using multiple views is that these are highly correlated sharing the same response pattern within the dictionary and thus this conciseness can enhance the overall ATR performance. In [19] authors suggest the $\mathrm{L}_{1 / 2}-\mathrm{NMF}$ technique that combines the $l_{1 / 2}$-norm optimization to identify the sparsest solution, with a Non-negative Matrix Factorization (NMF) scheme. The NMF features used as input to the SRC technique are the outcome of a NMF process that is applied on the SAR imagery. Dong et al. in [11] use the monogenic signal of a SAR image as an input to the SRC process. This signal comprises of the 2D SAR image signal and its Riesz transformed representation.

Deep Convolutional Neural Networks (CNNs) have also been suggested for SAR ATR. Literature proposes several CNN based solutions that use handcrafted CNNs [5], [8], [12], [13], [30] that are trained on SAR template images. Recently a 
Recurrent Neural Network is also suggested [31].

In the context of SAR ATR, SC and CNN based methods have individually shown theirs strengths by achieving quite high recognition rates. However, these techniques have not been fused yet such as to complement their strengths and afford an even higher recognition rate. Most important reasons to fuse SC and CNN based ATR are:

a. To extend the search space for the SAR ATR solution as SC and CNN ATR search for an ATR solution in different spaces. Indeed, SC ATR searches for linear projections between the target and the feature spaces while CNN ATR for non-linear projections. Hence, by fusing these two concepts, we essentially span a wider search area aiming a gaining higher ATR rates.

b. Combined classifiers can improve performance, as training a single classifier to work well for all test data is difficult. This multi-classifier strategy might not necessarily out-perform a single best performing classifier, but on average, it will perform better.

Driven by these reasons we fuse CNN and SC. Even though fusion in general, can be at a data, feature or decision level, in this paper we implement a decision level fusion. This is because the data modality for both contributing ATR modules, i.e. CNN and SC, is the SAR and therefore a data fusion scheme is not applicable. Additionally, despite feature-fusion could be an option, we neglected it as this would create an even larger feature encoding every SAR image, increasing the processing time needed to perform feature matching and neglecting it from military applications that require near-real time performance.

Additionally, state-of-the-art CNNs such as AlexNet [32], VGG [33], GoogleNet [34] and ResNet [35] have not been used in the context of SAR ATR. Driven by that, we suggest a novel architecture dubbed $l_{1-2} C C N N$ that fuses an adaptive $l_{1}$-norm, $l_{2}$-norm SC scheme with a modified Clustered AlexNet CNN (CCNN) that uses a multi-class Support Vectors Machine (SVM) structure for final classification. The contributions of this paper can be summarized as:

a. In contrast to current SC based applications that use a fixed $l_{p}-$ norm, we propose a novel adaptive elastic net type optimization that balances the advantages $l_{1}-$ norm and $l_{2}-$ norm depending on the characteristics of each scene SAR imagery. It is worth noting, that in contrast to current SC SAR ATR solutions, we neglect using the scattering centers of the SAR imagery in order to reduce the additional processing cost.

b. We extend the usability of the AlexNet CNN from the visual domain to the SAR by introducing a hidden layerclustering technique. This modification is combined with a multi-class SVM classification module that bridges the visualSAR modality gap.

c. We innovatively fuse these two multi-discipline solutions under a decision level scheme that adaptively changes its fusion weights. Fusing these two techniques aims at expanding the search region of the SAR ATR solution and overcome the weaknesses of each of the two techniques.

The rest of the paper is organized in the following sections.
Section II introduces the proposed $l_{1-2}-C C N N$ architecture, while Section III evaluates our method on the MSTAR dataset. Finally, Section IV concludes the paper.

\section{SAR ATR ARCHITECTURE}

The suggested architecture relies on a weighted SC, a clustered AlexNet variant and a decision level fusion scheme aiming at exploiting the advantages of all three techniques, each of which will be analyzed in the following paragraphs.

\section{A. Sparse Coding}

Sparse Representation or Coding aims at recovering a sparse representation $x$ of a measured 1-dimensional signal $y$ as a linear combination of a few atoms i.e. entries of a dictionary $D$ [11], [29], [36], [37]:

$$
y=D x \text { with } D \in \mathfrak{R}^{M \times N}, M<<N, \operatorname{Rank}\{D\}=M
$$

where $x \in \mathfrak{R}^{N \times 1}$ is a coefficient vector whose non-zero entries determine the linear combination of the atoms in $D$ that reconstruct measurement $y$. Ideally $x$ should be $K$-sparse with $K=1$, i.e. all entries to be zero except from the one that associates $y$ with the training sample within $D$.

Since $M<N$, Eq. (1) is underdetermined and therefore has infinite solutions. Determining the best solution $x_{b}$ is an optimization problem that is ideally solved using the $l_{0}-$ norm in order to identify the sparsest vector $x_{0}$ out of the infinite solutions:

$$
x_{b}=\arg \min \|x\|_{0} \text { subject to } D x=y
$$

where $\|x\|_{0}:=\#\left\{i: x_{i} \neq 0\right\} \leq N$, which counts the number of non-zero entries in $x$. Solving Eq. (2) is NP-hard and therefore compressive sensing theory [38] suggests exploiting the sparse nature of the signal $y$ (if it fulfills that prerequisite) and recovers the initial signal by solving the optimization problem:

$$
x_{b}=\arg \min \|x\|_{1} \text { subject to } D x=y
$$

For 2-dimensional data such as SAR images $I \in \mathfrak{R}^{a \times b}$, these are first remapped from the original $a \times b$ image basis to a $c \times d$ feature basis by down-sampling $I$ using bicubic interpolation. It is worth noting that $I$ is not the complex data representation of a SAR image, but a grayscale 2D image where the pixel values correspond to the amplitude of the SAR based reflectivity that is constrained in the $0-255$ value range. We down sample $I$ to reduce its dimensions and thus decrease the computational demands and increase the robustness of the SC ATR module to noise, resolution variation and to depression angle variation. We examine several down-sampling factors to identify the one that presents an optimum performance (Section III-B-1). The reason for exploiting bicubic interpolation rather than other interpolation techniques appropriate for 2D imagery in general, is smoothing $I$, which enhances robustness to nuisance factors, 
e.g. noise.

Then the remapped images are converted into a $m \times 1$ column vector with $M=c \times d$ [39] and are normalized to have a unit $l_{2}-$ norm:

$$
I \in \mathfrak{R}^{a \times b} \longrightarrow I_{s p} \in \mathfrak{R}^{M \times 1}, M=c \times d<<a \times b
$$

Finally, the dictionary is defined as $D=\left[D_{1}, D_{2}, \ldots, D_{j}\right]$ where $j$ is the number of training classes and each class is defined as $D_{o}=\left[I_{s p}^{o-1}, \ldots, I_{s p}^{o-k}\right] \in \mathfrak{R}^{m \times k}, o \leq j$ with $k$ the number of atoms/ entries per class $j$. Hence, we create an overcomplete dictionary $D \in \mathfrak{R}^{M \times N}$ with base elements the 1D SAR feature vectors of the corresponding SAR training images as created by Eq. (4). In contrast to current SC based SAR ATR methods [8], [11], [14], we do not create the 1D SAR feature vectors from pre-processed grayscale SAR images but from the raw grayscale SAR images. The advantage of using directly the grayscale SAR imagery is relaxing the complexity and thus reducing the processing burden of the proposed SC module without though sacrificing its SAR ATR performance (Section III). It is worth noting that the size of $D$ has a major influence on the performance of the SC algorithm. Specifically, $N$ purely depends on the available training images, but the value of $M$, i.e. 1D feature vector length, even though fixed it is user-defined, meeting the constraints presented in Eq. (1). For this work, we examine several feature lengths such as to optimize the SC SAR ATR performance (Section III-B-1).

$\mathrm{SC}$ classification relies on the assumption that a new unknown test image $I^{\prime}$ from class $u$ that is converted into a 1D feature vector $I_{s p}^{\prime}$ lies within the same subspace with the training atoms of the same class. Thus $I_{s p}^{\prime}$ can be represented by Eq. (1) and solved with Eq. (3). It is reminded that the test SAR image is not input directly to Eq. (3) but we exploit its corresponding $1 \mathrm{D}$ feature vector $I_{s p}^{\prime}$ that is produced according to Eq. (4).

Driven by the underlying SAR imagery data structure, we generalize [40] and consider that an $l_{1}-$ norm SC is effective for non-Gaussian type 1D SAR feature vectors, whereas $l_{2}-$ norm for Gaussian type. Therefore, given a test SAR image, we first remap it according to Eq. (4) and then analyze its core structure to identify if it is a Gaussian or a nonGaussian type. Specifically, we analyze the 1D SAR feature vector $I_{s p}^{\prime}$ as a combination of a two-component Gaussian Mixture Model (GMM) [41]:

$$
\begin{gathered}
p\left(I_{s p}^{\prime}\right)=\sum_{i=1}^{2} \varphi_{i} N\left(I_{s p}^{\prime} \mid \mu_{i}, \sigma_{i}\right) \\
\varphi_{1}+\varphi_{2}=1 \\
N\left(I_{s p}^{\prime} \mid \mu_{i}, \sigma_{i}\right)=\frac{1}{\sigma_{i} \sqrt{2 \pi}} \exp \left(-\frac{\left(I_{s p}^{\prime}-\mu_{i}\right)^{2}}{2 \sigma_{i}^{2}}\right)
\end{gathered}
$$

where $\mu_{i}, \sigma_{i}$ and $\varphi_{i}$ are the mean, the variance and the component weight of the $i^{\text {th }}$ GMM component of the 1D SAR feature $I_{s p}^{\prime}$.

Then, we substitute Eq. (3) with an elastic net regularization technique [42] that is extended to use an adaptive coefficient estimator such as to optimize the regression problem depending on the GMM 1D SAR feature vector analysis :

$$
x_{b}=\underset{x}{\arg \min }\left((1-a)\|x\|_{2}^{2}+a\|x\|_{1}\right) \text { subject to } D x=I_{s p}^{\prime}
$$

where $a$ is the penalty factor that we adaptively define as:

$$
a=\left|\frac{1-\max \left(\varphi_{l}\right)}{0.5}-\varepsilon\right|
$$

such as $a \rightarrow 1$ when $\max \left(\varphi_{i}\right) \rightarrow 0.5$ and $a \rightarrow 0$ when $\max \left(\varphi_{i}\right) \rightarrow 1$, with $\varepsilon$ a very small constant (in our trials we use $\varepsilon=10^{-5}$ ) and $\varphi_{i}$ the GMM component weight. We solve Eq. (8) using the Least Angle Regression - Elastic Net (LARS-EN) [42], with a convergence threshold of $10^{-4}$ for the cyclical coordinate descent [43] that is computed along the regularization path with $10^{5}$ maximum iterations. Using the LARS-EN solver implies the penalty factor to be in the range of $(0,1]$.

It is important to note that in contrast to [40], our extension:

a. Does not consider SC classification on 1D data that are affected by Gaussian and non-Gaussian noise. In our architecture, we solve Eq. (5) after analyzing the underlying structure of 2D SAR imagery and determine whether the core of this structure is governed by a Gaussian or a non-Gaussian distribution.

b. Does not involve a fixed value of the parameter $\alpha$ that is determined after a tuning process. Instead of that fixed approach, we adaptively estimate $a$ for each 2D SAR target image that depends on a GMM based analysis of the target image. The advantage of this adaptive estimation is that it fully exploits the capabilities of the elastic net solution of Eq. (5) as it spans $a$ to a range of possible values with $a \in(0,1]$.

This methodology aims at determining whether a single dominant Gaussian distribution can or cannot describe the 1D feature $I_{s p}^{\prime}$ of the SAR image $I^{\prime}$, and accordingly adapt Eq. (9) such as to optimize the elastic net given by Eq. (8). Fig.1 shows two extreme case examples where the 1D feature $I_{s p}^{\prime}$ of the SAR image $I^{\prime}$ (blue curve) is analyzed into a twocomponent GMM (black and red curve show the Gaussian distribution of each model). Depending on the contribution of each GMM, in Fig. 1(a) we show an example of a 1D feature vector that has two equally important Gaussian distributions and thus in Eq.(8) we input $a=0.922\left(\max \left(\varphi_{l}\right)=0.538\right)$, while in Fig. 1(b) an example of one dominant Gaussian distribution and hence in Eq.(8) we input $a=0.308$ ( $\left.\max \left(\varphi_{t}\right)=0.846\right)$. Therefore, in the former case of Fig. 1(a), the SC SAR ATR problem of Eq. (8) is solved mostly based on a $l_{1}-$ norm scheme while for the latter case of Fig. 1(b) the solution of Eq. (8) is more affected by the $l_{2}-$ norm contribution. 


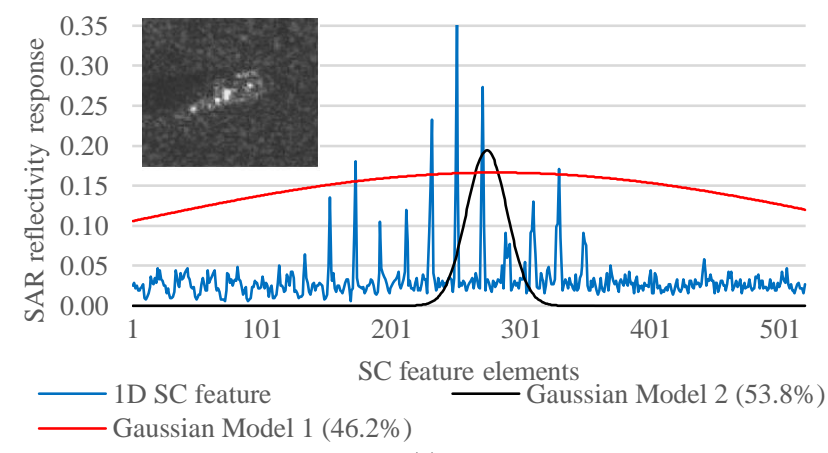

(a)

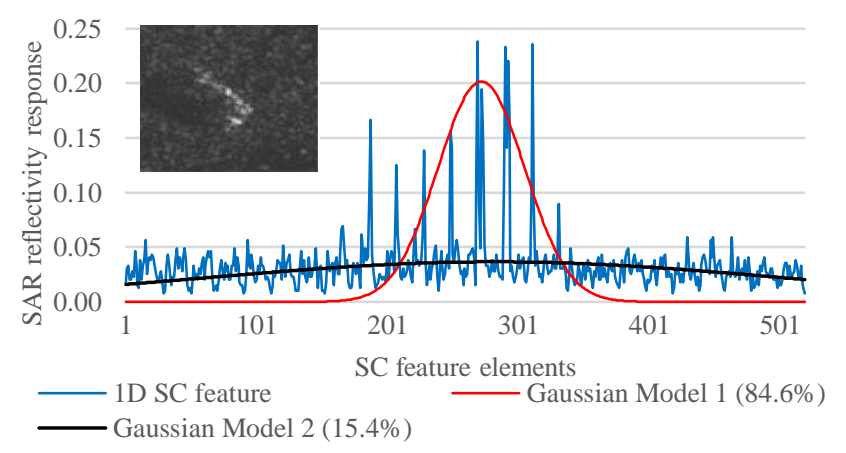

(b)

Fig. 1. GMM analysis of a SAR image (top left) in the 1D feature space showing (a) mutual GMM importance (b) dominant GMM importance (percentage highlights the influence per GMM distribution - GMMs scaled based on their \% contribution)

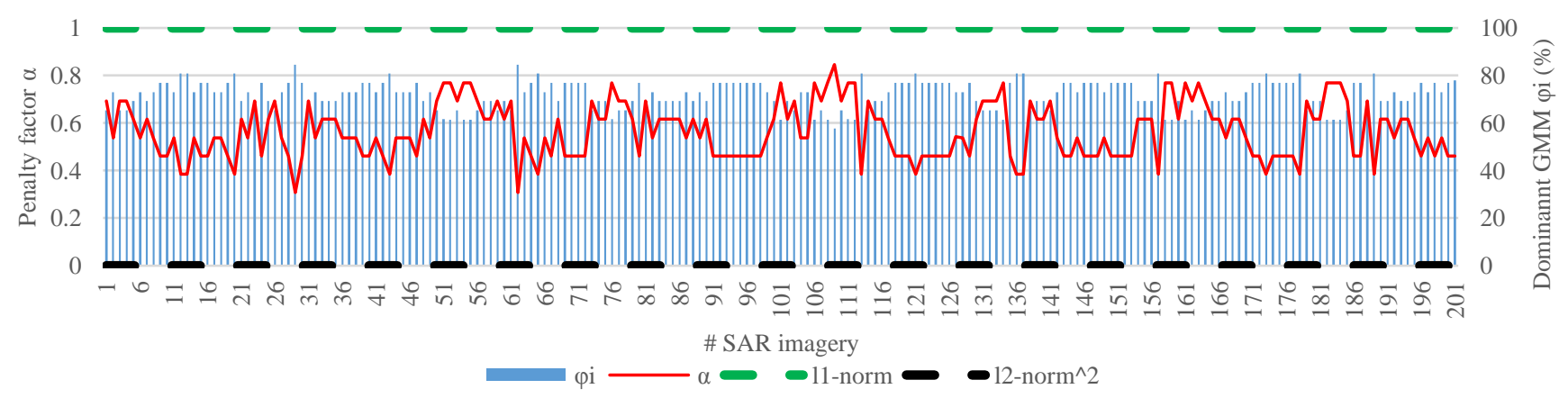

Fig. 2. Dependency of penalty factor $a$ with the dominant GMM distribution $\varphi_{i}$

(dashed lines show pure $\|x\|_{1}$ for $a=1$ and $\|x\|_{2}^{2}$ for $a=0$ solution schemes)

Fig. 2 shows the $\varphi_{i}$ variation of the dominant Gaussian component and the corresponding penalty factor $a$ over a few example SAR images. From Fig. 2 it is evident that the contribution $\varphi_{i}$ of the dominant GMM varies based on the SAR image reflectivity that affects the $1 \mathrm{D}$ feature vector $I_{s p}$, which in turn adaptively adjusts the penalty factor $a$ (Eq. (9)) and ultimately influences the elastic net regularization of Eq. (8). Fig. 2 also shows the two extreme cases where the 1D SAR feature vector is a perfect balance of two GMMs i.e. $\varphi_{1}=\varphi_{2}=0.5$ and thus $a=1$ and therefore from Eq. (5) the SC ATR problem for that SAR image is solved purely based on $l_{1}$-norm. Fig. 2 also shows a hypothetical perfect imbalance of the two GMMs with $\varphi_{1}=1$ and $\varphi_{2}=0$ (or vice versa). In that case, $a=\varepsilon$ and from Eq. (5) the SC ATR problem for that SAR image is solved based on a $l_{2}-$ norm scheme.

\section{B. Clustered Convolutional Neural Networks}

In the context of SAR ATR, literature suggests several CNN based solutions that rely on handcrafted CNNs [5], [7], [8], [12], [13]. A common feature of these CNN architectures is their relatively low depth that varies from six up to nine layers, opposing to the mainstream visual domain CNNs where layers are 23 for AlexNet [32], 16 or 19 for VGG [33] depending on the version, 22 for GoogleNet [34] and 152 for ResNet [35]. This is because visual images have a higher information content per pixel compared to the radar reflections presented in a SAR image. Unarguably, current mainstream CNNs have an exceptional classification capability in the visual domain. A typical way to deviate these CNNs from the dataset these were trained on, is by exploiting the Transfer Learning technique [44]. Nevertheless, this technique is not always effective in steering the weights of the CNN towards a completely different data modality i.e. from visual to SAR imagery [45]. Additionally, the limited number of publicly available military SAR imagery imposes the SAR ATR CNNs to populate the training images either by creating artificial variants e.g. rotated versions of the existing templates or by sampling patches out of the image. Opposing to that, RGB images are widespread and thus the pre-trained CNNs [32][35], [46]-[48] for that domain can exploit a massively larger training set.

Driven by the advantages of the RGB pre-trained CNNs we propose a multi-discipline and multi-modal architecture that combines the concepts of CNN and Multiclass Support Vector Machine (M-SVM) classification [49]. The intention is to transfer the already proven classification capability of the AlexNet [32] from the RGB domain to the X-band SAR without using Transfer Learning [44]. This is because the combination of the completely different data modality between SAR and visual imagery along with the lack of SAR training samples imposes a huge constrain to steer the weights of these CNNs towards SAR data and thus offering a moderate classification performance [45].

AlexNet is a 23-layered network that encapsulates from an RGB image features that vary from low-level corners and blobs, in the initial hidden layers, up to high-level RGB 
oriented complex features in the last layers. Although AlexNet is powerful, it has been trained on the RGB images of ImageNet [50] that are completely different to SAR imagery. AlexNet is trained on RGB color bands while SAR images contain radar reflections. Therefore, directly applying AlexNet on SAR imagery is not an optimum solution. Hence, we group the 23 layers of AlexNet into nine clusters $l$ of varying feature description capability, introducing the ClusteredAlexNet (C-AlexNet) presented in Table I. Notation $l$ refers to the cluster layer activated with $l \in\{1,2,3,4,5,6,7,8,9\}$. This means, for instance, $l=4$ activates up to AlexNet's clustered layer 4 while the remaining layers $\{5,6,7,8,9\}$ are discarded. C-AlexNet uses the same parameters (stride, padding and convolutional filter sizes) as in the original implementation [32].

This specific clustering scheme is directly related to the position of the convolutional layers within AlexNet, which in turn are directly linked to the complexity of the features extracted from each cluster. That is, the deeper the convolutional layer, the more complex and data specific the detected features are. It should be noted that a fully connected layer is a convolutional layer that uses a kernel that has the size of the output of the previous hidden layer [51]. Therefore, the input layer of clusters six to eight is a fully connected rather than a convolutional layer.

Given a SAR image $\mathrm{I}^{a \times b}, \quad a, b \in Z^{+} \quad$ and $I(s, t) \in\{0,1, \ldots, 255\}$ with $1 \leq s \leq a$ and $1 \leq t \leq b$, we initially remap $I$ into a 3-D tensor to meet the input requirements of AlexNet:

$$
I_{1}=B(I)\|B(I)\| B(I)
$$

where $B(\cdot)$ is a bicubic interpolation process and $\|(\cdot)$ is a 3D concatenation of a single SAR image in order to replicate the RGB layers that AlexNet requires as an input.

Once $I_{1}$ is input to the C-AlexNet, it is transformed into a 3D tensor $X^{l} \in \mathfrak{R}^{H^{l} x W^{l} x D^{l}}$ which propagates through the hidden layers until it becomes the output $Y^{l}$ of the end-layer of cluster $l$. Hence, $X^{1}$ is the input to cluster $l=1, X^{2}$ is the output of cluster $l=1$ and simultaneously the input to $l=2$ etc. Notation $H^{l}, W^{l}$ and $D^{l}$ refer to the height, width and depth of the tensor at clustered layer $l$ and an element belonging to

TABLE I

Clustered AleXNet LAYERS

\begin{tabular}{cll}
\hline \hline $\begin{array}{r}\text { C-AlexNet } \\
\text { layer ID }(l)\end{array}$ & $\begin{array}{c}\text { AlexNet } \\
\text { layer ID }\end{array}$ & \multicolumn{1}{c}{ Operations involved } \\
\hline 1 & $1-2-3-4-5$ & $\begin{array}{l}\text { Image input - Convolution - ReLU - } \\
\text { Normalization - Max pooling } \\
\text { Convolution - ReLU - Normalization - } \\
\text { Max pooling }\end{array}$ \\
2 & $6-7-8-9$ & $\begin{array}{l}\text { Convolution - ReLU } \\
\text { Convolution - ReLU }\end{array}$ \\
3 & $10-11$ & Convolution - ReLU - Max pooling \\
4 & $12-13$ & Fully connected - ReLU \\
5 & $14-15-16$ & Fully connected - ReLU \\
6 & $17-18$ & Fully connected \\
7 & $19-20$ & SoftMax - Classification output \\
8 & 21 & \\
9
\end{tabular}

$X^{l}$ has an index set of $\left(u^{l}, v^{l}, d^{l}\right)$ with $0 \leq u^{l} \leq H^{l}$, $0 \leq v^{l} \leq W^{l}, 0 \leq d^{l} \leq L^{l}$. Network activations are computed by forward propagating input $I_{1}$ through the $\mathrm{CNN}$ architecture up to the specified layer $l$. For the feedforward process we use a mini batch size of one i.e. one training instance per iteration to estimate the gradient of the loss function and estimate the response of the CNN network. The reasoning of choosing a mini batch size of one is to increase the accuracy of the response.

3D tensors $X^{l}$ and $Y^{l}$ are stacks of $2 \mathrm{D}$ matrices that highlight features of various complexity in a response map type of representation. As the $X^{l}$ tensor propagates within the CNN's activated clusters and ultimately becomes tensor $Y^{l}$, the tensor's size changes based on the size of the convolutional kernel of each layer. That is a kernel size of $11 \times 11 \times 3$ for cluster 1 , the height and width of which approximately halves for each subsequent convolution till cluster 3 and thereafter it stabilizes at a kernel size of $3 \times 3$ (height $\mathrm{x}$ width). Tensors $X^{l}$ and $Y^{l}$ can be regarded as a generalized scale-space theory [52] concept where the various scales are envisaged via the subsequent shrinking of the convolutional kernel size and the octaves via the kernel weights that are auto-adjusted by the CNN during the training stage. In computer vision, scale-space is an important theory for keypoint detection contributing to the robustness of pattern recognition algorithms. Therefore, by linking tensors $X^{l}, Y^{l}$ with scale-space theory, we highlight the importance of these tensors and validate their contribution in regards to pattern recognition tasks as examined in this paper.

As noted in Table I, the features that further propagate in our clustered SAR ATR architecture are the ones provided by the end-layer of each clustered layer $l$ that may be a Rectified Linear Unit (ReLU) layer, a Max Pooling layer or a Fully Connected layer. Therefore, it is important to present the operating details of these layers.

\section{1) $\operatorname{ReLU}$}

This layer increases the non-linearity of a CNN by applying an individual truncation process on every $X^{l}\left(u^{l}, v^{l}, d^{l}\right)$ :

$$
Y_{u, v, d}^{l}=\max \left\{0, X_{u, v, d}^{l}\right\}
$$

where $Y_{u, v, d}^{l}$ is the output of the $l$ cluster layer. The advantages of ReLU against the classic tanh activation function are the reduction in training time [32] and incorporating a purely supervised training scheme avoiding the need of unsupervised pre-training [53].

\section{2) Max Pooling}

This operation substitutes a sub-region $X_{s}^{l}$ of size $s \mathrm{X} s$, i.e. pooling size, of the tensor $X_{u, v, d}^{l}$ with its maximum value:

$$
Y_{u, v, d}^{l}=\max \left(X_{s}^{l}\right)
$$


$Y_{u, v, d}^{l}$ will have a size of $H^{l+1}=H^{l} / s, W^{l+1}=W^{l} / s$ and $D^{l+1}=D^{l}$.

\section{3) Fully Connected}

Through the fully connected layer, the $X^{l}\left(u^{l}, v^{l}, d^{l}\right)$ input of size $H^{l} \times W^{l} \times D^{l}$ is remapped to:

$$
Y^{l}=w_{u, v, d}^{l} X^{l}+\text { bias }
$$

that has size $H_{o}^{l} \times W^{l} \times D^{l}$, where $w_{u, v, d}^{l}$ is the weight parameter that the fully connected layer is aiming at tuning and $H_{o}^{l}$ the height of $Y^{l}$ that is defined during the design of the convolutional neural network.

In the suggested architecture, the output tensor $Y_{u, v, d}^{l}$ of the $l$ cluster layer is remapped into a $1 \mathrm{D}$-feature vector of length $u^{l} \times v^{l} \times d^{l}$ by undergoing a multi-feature fusion process. The latter is implemented via a multi-dimensional vectorization process defined as:

$$
\underset{u=1, v=1}{H, W}\left(a_{u, v, d}\right):=\left[a_{1,1, d}, \ldots, a_{H, 1, d}, a_{2,1, d}, \ldots, a_{H, 2, d}, a_{1, W, d}, \ldots, a_{H, W, d}\right]^{T}
$$

over dimension $d$ which is then followed by a vectorization procedure:

$$
y^{l}=\operatorname{vec}\left(\underset{u=1, v=1}{\stackrel{H, W}{\Theta}}\left(Y_{u, v, \omega}^{l}\right)\right)
$$

where $\omega=[1, \ldots, d]$. The advantage of this multi-fusion process is encompassing both the feature responses and the topology of the features for the entire tensor depth.

\section{4) Multi-class Support Vector Machines (M-SVM)}

The $y^{l}$ feature produced from the C-AlexNet at layer $l$ is then used to train a one-vs-all M-SVM classification scheme. Given $j$ the number of classes, the $g^{\text {th }}$ class is trained with all the examples in the $g^{\text {th }}$ class having positive labels and the remaining classes having negative labels. For $h$ training images, the data $y^{l}$ vs. target class $\mathrm{Cl}$ correlation is $\left(y_{1}^{l}, C l_{1}\right), \ldots\left(y_{p}^{l}, C l_{p}\right), \ldots,\left(y_{h}^{l}, C l_{h}\right)$ with $C l_{p}=\{1, . ., j\}$ being the class of $y_{p}^{l}$. M-SVM performs multiple binary SVM classification tasks and labels the $y^{l}$ feature belonging to the class that gains the highest response. For a detailed analysis on SVM classification, the reader is referred to [54].

\section{Decision level fusion}

The 1D vector $x_{b}$ obtained from Eq. (8) includes responses from all the atoms within $D$ regardless of the class these belong. Thus, we remap $x_{b}$ to facilitate a single normalized response per target class $j$ given by:

$$
r_{p}^{S C}=\frac{x_{p}}{\max \left(x_{p}\right)}
$$

with $x_{p}$ a subset of $x_{b}$ that includes only the responses of the target class $p, p \leq j$.

Similarly, the output $Y^{l}$ of the activated layer in the clustered CNN module is converted into $r_{p}^{C N N}$ so that each target class has a single response:

$$
r_{p}^{C N N}=\left\|y^{l}\right\|_{\infty}
$$

Then we normalize the response per target class obtained from the suggested SC and C-AlexNet modules, i.e. $r_{p}^{S C}$ and $r_{p}^{C N N}$ respectively, to make them comparable. Normalization is done via the z-score technique and the SAR ATR decisionmaking function $\mathfrak{I}$ is based on a weighted winner takes it all concept that is given by:

$$
\mathfrak{I}=\underset{p}{\arg \max }\left(\lambda \frac{r_{p}^{C N N}-\overline{r_{p}^{C N N}}}{\sigma\left(r_{p}^{C N N}\right)} \| \frac{r_{p}^{S C}-\overline{r_{p}^{S C}}}{\sigma\left(r_{p}^{S C}\right)}\right)
$$

where $\|(\cdot)$ is a $1 \mathrm{D}$ concatenation process, $\bar{r}, \sigma(r)$ are the average and standard deviation of the corresponding SAR responses and $\lambda$ is a regulating parameter:

$$
\lambda=\left\{\begin{array}{cc}
1 & \text { if }\left|S_{I}-\bar{S}_{\text {template }}\right|<E \cdot \sigma\left(S_{\text {template }}\right) \\
1.25 & \text { otherwise }
\end{array}\right.
$$

with $S_{I}$ the target SAR image entropy, $E$ a tuning parameter while $\bar{S}_{\text {template }}$ and $\sigma\left(S_{\text {template }}\right)$ the average and standard deviation of the entropy of the templates. The role of parameter $\lambda$ is to tune finely the decision-making function of Eq. (19) depending on the deviation of the target's SAR image disorder $S_{I}$ in comparison to the disorder of the templates. The value of $\lambda=1.25$ is determined experimentally.

Our proposed Convolutional Neural Network and Sparse Coding data fusion architecture named $l_{1-2}-C C N N$ is presented in Fig. 3.

\section{EXPERIMENTS}

\section{A. MSTAR dataset}

We evaluate the performance of the proposed architecture on the MSTAR database [55], which includes the ground target classes presented in Fig. 4. Each class contains chips of $15^{\circ}$ and $17^{\circ}$ depression angles using an X-band SAR sensor, while some classes contain additional $30^{\circ}$ and $45^{\circ}$ depression angle viewings. All target SAR chips cover a full $0^{\circ}-360^{\circ}$ azimuth orientation. Table II presents the number of targets per type and depression angle used in this paper. To avoid the influence of background, we crop all images by extracting a $80 \times 80$ patch set at the center of the image. For compatibility with current literature we adopt [55] and establish a training set based on the 17 . 


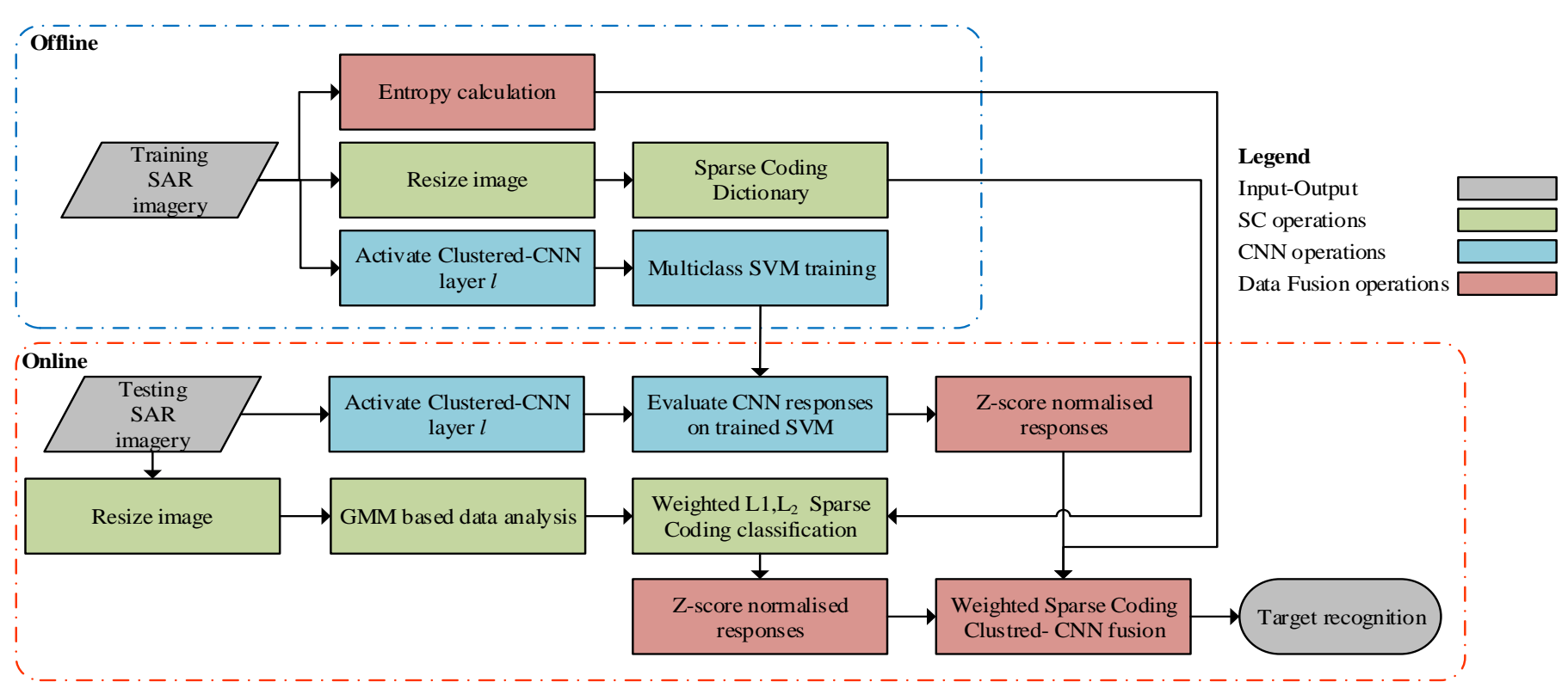

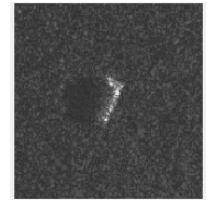

2S1

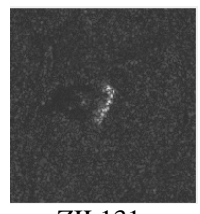

ZIL131

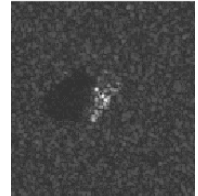

BMP2

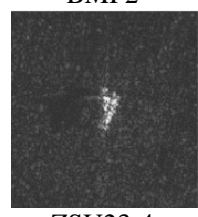

ZSU23-4

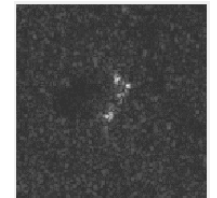

BRDM2

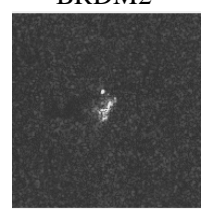

D7

(a)

Fig. 3. Proposed $\overline{l_{l-2}-} \bar{C} C \overline{N N}$ architecture for $\overline{\mathrm{S}} \mathrm{AR}$ ATR

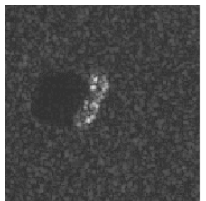

BTR60

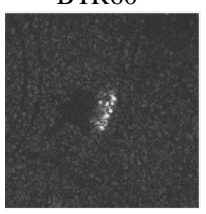

T62

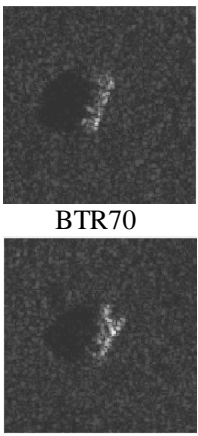

T72

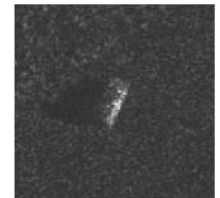

$15^{\circ}$

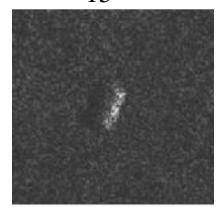

$30^{\circ}$

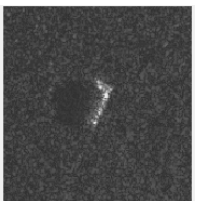

$17^{\circ}$

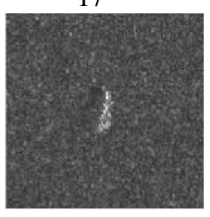

$45^{\circ}$

Fig. 4. (a) 10 classes of the public MSTAR database at $17^{\circ}$ depression angle (b) the $2 \mathrm{~S} 1$ target at various depression angles while at same azimuth

TABLE II

MSTAR DATABASE

\begin{tabular}{|c|c|c|c|c|c|c|c|c|c|c|c|c|c|c|c|c|}
\hline Target & & BMP2 & & BTR70 & & $\mathrm{T} 72$ & & BTR60 & $2 \mathrm{~S} 1$ & BRDM2 & D7 & $\mathrm{T} 62$ & ZIL131 & ZSU23/4 & \multicolumn{2}{|c|}{ Sum } \\
\hline $\operatorname{train} 17^{\circ}$ & 233 & 232 & 233 & 233 & 232 & 231 & 228 & 256 & 299 & 298 & 299 & 299 & 299 & 299 & $\begin{array}{c}\text { SOC-1 } \\
2747\end{array}$ & $\begin{array}{c}\text { SOC-2 } \\
3671\end{array}$ \\
\hline
\end{tabular}

\section{B. 3-class problem}

We use this experiment to fine-tune the free parameters of our architecture i.e. the modules of SC, C-Alexnet and decision level fusion. The target classes used are the BMP2, T72 and BTR70. For the former two we use all three variants namely the 9563,9566 and $\mathrm{c} 21$, for the T72 the 132, 812 and s7 and for BTR70 the c71 which is the only one included in the dataset. Images captured at $17^{\circ}$ depression angle are used as training and images at $15^{\circ}$ for testing.

Specifically, our architecture is governed by the feature dimension $m$ of the adaptive $l_{1-2}$-norm SC (Eq. (8)), the layer $l$ of the C-AlexNet that is activated (Table I) and the entropy boundary $E$ (Eq. (19)) during the fusion stage. During tuning, we set as baseline values $m=512, l=2$ and $E=3$, and evaluate the 3-class ATR performance of the suggested technique by altering consecutively one of these three values. For the given baseline parameters, Table III highlights the performance of our architecture compared to current algorithms. It is evident that fusing the SC and CNN techniques under their suggested modified versions can outperform solutions that rely on a single method only. All trials are performed in MATLAB on an Intel i7 with 16GB RAM and an Nvidia Quadro K2200 GPU processor. MatConvNet [56] is used to implement AlexNet. The value of $\lambda=1.25$ in Eq. (19) does not affect the performance of the 3-class ATR problem.

\section{1) Adaptive $L_{p}$-norm based SC optimization}

We create and evaluate a dictionary $D \in \mathfrak{R}^{d \times 1622}$ of various feature dimensions $d=\{64,128,256,512,1024\}$. As expected, Fig. 5 (a) shows that the larger the feature space dimension the 
TABLE III

3-CLASS ATR (\%)

\begin{tabular}{|c|c|c|c|c|c|c|c|c|c|c|c|}
\hline & CM [57] & $\mathrm{BMO}$ [23] & SRF [22] & Huang's [21] & DFSS [58] & ASC [24] & PCA [59] & 2DPCA [60] & $\begin{array}{c}l_{1-2-}-\mathrm{SC} \\
\text { only }\end{array}$ & $\begin{array}{c}\text { CCNN } \\
\text { only }\end{array}$ & $\begin{array}{c}l_{1-2^{-}} \\
\mathrm{CCNN}\end{array}$ \\
\hline BMP2 & - & 97.28 & 94.89 & 94.38 & 91.65 & 97.27 & 97.44 & 99.15 & 94.90 & 98.30 & 98.5 \\
\hline BTR70 & - & 98.98 & 96.43 & 98.47 & 99.48 & 97.96 & 99.49 & 98.47 & 97.40 & 98.48 & 100 \\
\hline $\mathrm{T} 72$ & - & 97.78 & 96.91 & 96.91 & 96.04 & 97.53 & 95.92 & 98.45 & 96.90 & 99.14 & 99.50 \\
\hline Avg. & 98.69 & 97.58 & 95.98 & 96.04 & 95.72 & 97.58 & 97.61 & 98.75 & 96.40 & 98.64 & 99.33 \\
\hline
\end{tabular}

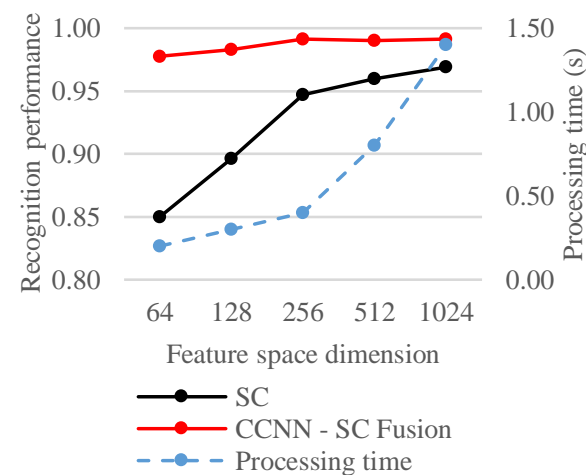

(a)

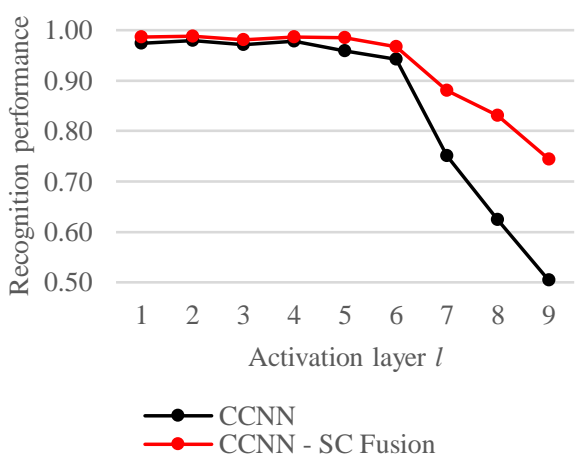

(b)

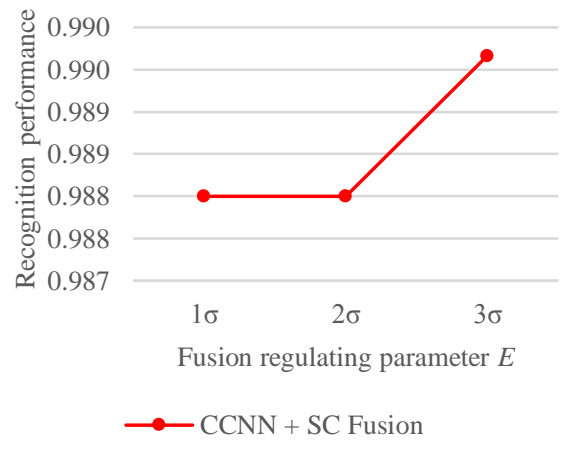

(c)

Fig. 5. Tuning parameters (a) SC feature space dimension (b) $C C N N$ activation layer (c) $E$ decision level fusion regulating value

better the classification performance but the greater the processing time. Fig. 5 (a) shows that for the chosen feature length size of $m=512$, the total processing time for the fused SAR ATR solution we propose is $800 \mathrm{~ms}$. This is because by increasing the feature space dimension, the SC based encryption becomes more distinct but Eq. (8) requires more processing time to provide a solution.

\section{2) C-AlexNet activation layer optimization}

During this tuning phase, we vary the activating layer of the CCNN according to Table I. Fig. 5 (b) shows that the deeper the activated layer the more RGB specific the feature response becomes and harder to steer the CNN towards the SAR data domain. Thus the less capable the M-SVM is to linearly separate the three target classes in the activated feature space. Optimum performance for the suggested fused scheme is identified at $l=2$ achieving $99.0 \%$ target recognition.

\section{3) Decision level fusion optimization}

We investigate how the decision level fusion regulating parameter $E$ affects the overall performance of our proposed SAR ATR architecture. From Fig. 5 (c) it is evident that this parameter has a minor role to the overall performance but it can still affect it.

\section{Assessment against large depression variation}

For this trial, we use three similar targets, namely the $2 \mathrm{~S} 1$, the BRDM2 and the ZSU 23-4. Images at $17^{\circ}$ depression angle are used for training, while the $15^{\circ}$, and $30^{\circ}$ and $45^{\circ}$ for testing. Table IV shows that the suggested multi-discipline scheme affords a high performing ATR solution.

Depression variation involves a non-linear feature transformation and since our $l_{1-2}$-norm solution seeks for linear projections from the image space to the feature space the low performance of the SC module is anticipated [39].

\section{Assessment against resolution variation}

We challenge the robustness of the $l_{1-2}-C C N N$ to resolution variations from $0.3 \mathrm{~m} \times 0.3 \mathrm{~m}$ (original resolution), down to $0.7 \mathrm{~m} \times 0.7 \mathrm{~m}$. Table $\mathrm{V}$ shows a target under these resolutions along with the performance of the suggested technique and the performance of current algorithms.

Table $\mathrm{V}$ shows that $l_{1-2}-C C N N$ outperforms all competitor solutions, while at the lowest resolution it still manages a $94.77 \%$ recognition rate. The robustness of $l_{1-2}-C C N N$ originates from the robustness of its individual modules i.e. the $l_{1-2}-S C$ and $C C N N$, which rely on the low-level abstract features extracted from the $l=2$ layer of C-AlexNet and adaptive $l$-norm process of the $l_{1-2}-\mathrm{SC}$ as described in Section II-A.

\section{E. 10-class ATR}

Literature suggests various target configurations for the 10class ATR problem, with commonly used the standard operation conditions 1 (SOC-1) and SOC-2. Although both are 10-class ATR subsets, their difference relates to the variants of BMP2 and T72 used. Specifically, SOC-1 for both training and testing includes only serial number 9563 for BMP2 and only serial number 132 for T72. SOC-2 uses all available serial numbers for both targets, for training and testing. Both SOC-1 and SOC-2 ATR evaluated based on the target's class and not its serial number. For both target set configurations, the $17^{\circ}$ depression angle is used for training and the $15^{\circ}$ for testing.

Tables VI and VII compare the ATR performance of $l_{1-2-}$ $C C N N$ against current literature for the corresponding SOC-1 
TABLE IV

3-CLASS ATR WITH LARGE DEPRESSION VARIATION (\%)

\begin{tabular}{ccccccc|ccc}
\hline \hline & NNSC [19] & L $1 / 2-$ NMF [19] $^{*}$ MSRC [11] & CKLR2 [37] & $\begin{array}{c}\text { Bayesian CS } \\
{[14]}\end{array}$ & JSRC [29] & $l_{1-2}$-SC only & CCNN only & $l_{1-2}$-CCNN \\
\hline $15^{\circ}$ & 96.11 & 98.91 & - & 99.75 & 99.20 & 99.50 & 95.60 & 100 \\
$30^{\circ}$ & 87.25 & 91.42 & 98.49 & 98.29 & 89.60 & 91.80 & 77.30 & 99.43 & $\mathbf{9 9 0}$ \\
$45^{\circ}$ & 70.19 & $\mathbf{7 8 . 4 4}$ & 59.63 & 74.56 & 70.80 & 75.30 & 60.00 & 68.67 & 70.87 \\
\hline \hline
\end{tabular}

TABLE V

3-CLASS ATR WITH RESOLUTION VARIATION

\begin{tabular}{|c|c|c|c|c|c|}
\hline Resolution variation & $0.3 \mathrm{~m} \times 0.3 \mathrm{~m}$ (original) & $0.4 \mathrm{~m} \times 0.4 \mathrm{~m}$ & $0.5 \mathrm{~m} \times 0.5 \mathrm{~m}$ & $0.6 \mathrm{~m} \times 0.6 \mathrm{~m}$ & $0.7 \mathrm{~m} \times 0.7 \mathrm{~m}$ \\
\hline Example & 47 & & & & 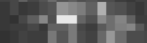 \\
\hline ASC & 97.58 & 96.50 & 93.90 & 91.50 & 85.90 \\
\hline $\mathrm{NMF}$ & 96.00 & 94.80 & 92.00 & 88.00 & 84.50 \\
\hline EFS & 94.00 & 90.00 & 84.00 & 76.00 & 68.00 \\
\hline Zernike & 96.00 & 92.00 & 86.00 & 82.00 & 74.00 \\
\hline PCA & 95.00 & 94.00 & 91.00 & 87.00 & 84.00 \\
\hline$l_{1-2}-\mathrm{SC}$ only & 96.40 & 93.68 & 94.24 & 85.05 & 91.66 \\
\hline CCNN only & 98.64 & 98.22 & 95.50 & 94.48 & 91.45 \\
\hline$l_{1-2}$-CCNN & 99.33 & 99.32 & 97.78 & 93.63 & 94.77 \\
\hline
\end{tabular}

TABLE VI

10-CLASS ATR ON SOC-1 (\%)

\begin{tabular}{ccccccccc|cc}
\hline \hline method & Chen's [8] & $\begin{array}{c}\text { MtCS } \\
{[28]}\end{array}$ & $\begin{array}{c}\text { Bayesian } \\
\text { CS [14] }\end{array}$ & $\begin{array}{c}\text { SAE } \\
{[18]}\end{array}$ & $\begin{array}{c}\text { DNN } \\
{[15]}\end{array}$ & $\begin{array}{c}\text { A-ConvNet } \\
{[5],[30]}\end{array}$ & $\begin{array}{c}\text { AdaGrad } \\
{[7]}\end{array}$ & SGD [7] & $l_{1-2}$-SC only & CCNN only \\
\hline avg $(\%)$ & 84.70 & 84.00 & 92.60 & 95.40 & 96.00 & 99.1 & 97.4 & 97.1 & 98.20 & 96.65 \\
\hline \hline
\end{tabular}

TABLE VII

10-CLASS ATR ON SOC-2 (\%)

\begin{tabular}{cccccccccccccccc}
\hline \hline method & DNN & IGT & Morgan's & BMO & KM & ASC & EFS Zernike & PCA & NMF Wagner's & DCNN & \multirow{2}{*}{ ZNC only } & CCNN only & $l_{1-2}$-CCNN \\
& {$[15]$} & {$[61]$} & {$[13]$} & {$[23]$} & {$[20]$} & {$[24]$} & {$[62]$} & {$[63]$} & {$[59]$} & {$[19]$} & {$[64]$} & {$[6]$} & $l_{1-2}$-SC & \\
\hline avg (\%) & 95.00 & 95.00 & 92.30 & 95.74 & 84.58 & 95.41 & 94.10 & 93.46 & 90.24 & 93.76 & $\mathbf{9 9 . 5 0}$ & $\mathbf{9 9 . 5 0}$ & 97.83 & 96.19 & $\mathbf{9 9 . 5 0}$ \\
\hline \hline
\end{tabular}

and SOC-2 MSTAR subsets. In both cases, the suggested $l_{1-2^{-}}$ $C C N N$ achieves top ATR performance, which is $99.86 \%$ for SOC-1 and $99.50 \%$ for SOC-2. In addition, Fig. 6 shows the corresponding confusion matrix per SOC subset. For better readability, we present only the confusion matrix of $l_{1-2^{-}}$ CCNN.

\section{F. 10-class ATR at various noise levels}

In this trial, we evaluate the robustness of current proposals to various noise levels. Trials are on the SOC-1 subset and the noise simulation is consistent with [5], [11] i.e. we randomly select a percentage of pixels in the target scene and replace their values with samples generated from a uniform distribution. It should be noted that template images both for the SC and CNN module are the original ones. Table VIII presents the performance achieved for noise levels varying from $1 \%$ up to $15 \%$. From Table VIII it is evident that the $l_{1-2^{-}}$ norm $\mathrm{SC}$ is extremely robust to noise levels due to its adaptive nature. Therefore, the $l_{1-2}-C C N N$ via its effective decision fusion process takes advantage of the high performing $l_{l-2-}$ norm SC module and outperforms with a great margin current solutions on SOC-1 with additive noise.

\section{G. Extending to other CNNs}

From all trials it can be concluded that $l_{1-2}-S C$ and $C C N N$ perform equally well for the 3-class and 10-class scenarios that do not have nuisance factors. The advantage of the fused
$l_{1-2}-C C N N$ is apparent because it preserves and even increases in quite a few cases, the robustness of $C C N N$ in the depression angle variation scenario and of $l_{1-2}-S C$ in the additive noise scenarios.

Driven by the results achieved, we extend our layerclustering strategy to VGG-16, GoogleNet and ResNet CNNs by utilizing their MatConvNet [56] implementations. As a reminder, similarly to AlexNet, all three CNNs are pre-trained on ImageNet [50]. The clustering methodology is similar to the one used for C-AlexNet, i.e. we cluster their layers so that the first layer of a cluster is a convolutional and the last layer is either a pooling or a ReLU layer. Based on the tuning process of Section III-B the optimum activation layer for the Clustered-VGG-16 (C-VGG-16) is $l=2$ that ends with the MaxPool_2 layer, while for the Clustered-GoogleNet (CGoogleNet) is $l=2$ that ends with the Pool_2 layer. Finally, the Clustered-ResNet (C-ResNet) is $l=3$ that ends with the res $2 a \_b r a n c h 2 b$ layer.

The first comparison among the clustered CNNs is on the SOC-1. Table IX shows that all clustered CNN variants perform equally well with $\mathrm{C}-\mathrm{VGG}$ offering the lowest processing time per scene image, $\mathrm{C}$-ResNet the highest CCNN ATR performance and C-AlexNet the smallest template storage requirement. Even though all $\mathrm{CNNs}$ perform quite well, C-AlexNet achieves the highest overall ATR performance fully exploiting the $\mathrm{SC}-\mathrm{CCNN}$ fusion scheme. 


\begin{tabular}{|c|c|c|c|c|c|c|c|c|c|c|}
\hline & $\overline{\vec{n}}$ & $\sum_{m}^{a}$ & 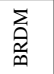 & $\begin{array}{l}\text { Oे } \\
\stackrel{\leftrightarrow}{\rightleftarrows}\end{array}$ & 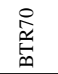 & $\hat{a}$ & 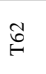 & Ê & $\stackrel{\mathrm{N}}{\mathrm{A}}$ & 胥 \\
\hline $2 \mathrm{~S} 1$ & 99.27 & 0 & 0.36 & 0 & 0.36 & 0 & 0 & 0 & 0 & 0 \\
\hline BMP2 & 0 & 100 & 0 & 0 & 0 & 0 & 0 & 0 & 0 & 0 \\
\hline BRDM & 0 & 0 & 100 & 0 & 0 & 0 & 0 & 0 & 0 & 0 \\
\hline BT R60 & 0 & 0 & 0 & 100 & 0 & 0 & 0 & 0 & 0 & 0 \\
\hline BTR70 & 0 & 0 & 0 & 0 & 100 & 0 & 0 & 0 & 0 & 0 \\
\hline D7 & 0 & 0 & 0.36 & 0 & 0 & 99.27 & 0 & 0 & 0.36 & 0 \\
\hline T62 & 0 & 0 & 0 & 0 & 0 & 0 & 100 & 0 & 0 & 0 \\
\hline $\mathrm{T} 72$ & 0 & 0 & 0 & 0 & 0 & 0 & 0 & 100 & 0 & 0 \\
\hline ZIL & 0 & 0 & 0 & 0 & 0 & 0 & 0 & 0 & 100 & 0 \\
\hline $\mathrm{ZSU}$ & 0 & 0 & 0 & 0 & 0 & 0 & 0 & 0 & 0 & 100 \\
\hline
\end{tabular}

(a)

\begin{tabular}{|c|c|c|c|c|c|c|c|c|c|c|}
\hline & $\overline{\text { जे }}$ & $\sum_{\infty}^{a}$ & 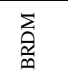 & 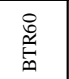 & 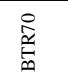 & $\hat{\theta}$ & త్ర & $\tilde{E}$ & $\overrightarrow{\mathrm{N}}$ & 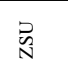 \\
\hline $2 \mathrm{~S} 1$ & 98.91 & 0.73 & 0 & 0 & 0.36 & 0 & 0 & 0 & 0 & 0 \\
\hline BMP2 (9563) & 0.51 & 98.98 & 0 & 0 & 0 & 0 & 0 & 0.51 & 0 & 0 \\
\hline BMP2 (9566) & 0 & 98.47 & 0 & 0.51 & 0.51 & 0 & 0 & 0.51 & 0 & 0 \\
\hline BMP2 (c21) & 0.51 & 98.98 & 0 & 0 & 0.51 & 0 & 0 & 0 & 0 & 0 \\
\hline BRDM & 0 & 0 & 100.00 & 0 & 0 & 0 & 0 & 0 & 0 & 0 \\
\hline BTR60 & 0 & 0 & 0.51 & 99.49 & 0 & 0 & 0 & 0 & 0 & 0 \\
\hline BTR70 & 0 & 0 & 0 & 0 & 100.00 & 0 & 0 & 0 & 0 & 0 \\
\hline D7 & 0.36 & 0 & 0.73 & 0 & 0 & 98.91 & 0 & 0 & 0 & 0 \\
\hline T62 & 0 & 0 & 0 & 0 & 0 & 0 & 99.63 & 0.37 & 0 & 0 \\
\hline $\mathrm{T} 72(132)$ & 0 & 0 & 0 & 0 & 0 & 0 & 0 & 100.00 & 0 & 0 \\
\hline $\mathrm{T} 72(812)$ & 0 & 0 & 0 & 0 & 0 & 0 & 0 & 100.00 & 0 & 0 \\
\hline T72 (s7) & 0 & 0 & 0 & 0 & 0.52 & 0 & 0 & 99.48 & 0 & 0 \\
\hline ZIL & 0 & 0 & 0 & 0 & 0 & 0 & 0 & 0 & 100.00 & 0 \\
\hline $\mathrm{ZSU}$ & 0 & 0 & 0 & 0 & 0 & 0 & 0 & 0 & 0 & 100.00 \\
\hline
\end{tabular}

(b)

Fig. 6. Confusion matrices (\%) of $l_{l-2} C C N N$ (a) SOC-1 (b) SOC-2 (rows are input classes and columns output classes)

TABLE VIII

10-CLASS SOC-1 ATR WITH AT VARIOUS NOISE LEVELS

\begin{tabular}{|c|c|c|c|c|c|}
\hline Gaussian noise level & $0 \%$ & $1 \%$ & $5 \%$ & $10 \%$ & $15 \%$ \\
\hline \multicolumn{6}{|l|}{ Example } \\
\hline A-ConvNet [5], [30] & 99.13 & 91.76 & 88.52 & 75.84 & 54.68 \\
\hline$l_{1-2}-\mathrm{SC}$ only & 98.24 & 95.68 & 95.38 & 95.42 & 94.51 \\
\hline CCNN only & 99.65 & 95.81 & 92.55 & 77.78 & 60.32 \\
\hline$l_{1-2}-\mathrm{CCNN}$ & 99.86 & 98.68 & 96.53 & 92.01 & 87.43 \\
\hline
\end{tabular}

TABLE IX

PERFORMANCE ANALYSIS OF CCNN VARIANTS ON 10-CLASS SOC-1 ATR

\begin{tabular}{ccccc}
\hline \hline Method & C-VGG & C-GoogleNet & C-AlexNet & C-ResNet \\
\hline $\begin{array}{c}\text { CCNN storage } \\
\text { (KB/template) }\end{array}$ & 186.6 & 602.1 & $\mathbf{4 3 . 3}$ & 200.1 \\
$\begin{array}{c}\text { CCNN time } \\
\text { (ms/image) }\end{array}$ & $\mathbf{6 . 3 5}$ & 14.65 & 17.50 & 45.9 \\
CCNN only (\%) & 97.86 & 95.08 & 96.65 & $\mathbf{9 8 . 0 7}$ \\
$l_{1-2}$-CCNN (\%) & 99.58 & 99.53 & $\mathbf{9 9 . 8 6}$ & 99.57 \\
\hline \hline
\end{tabular}

We continue our trials by evaluating the ATR performance for the 3-class recognition case of Section III. Table $\mathrm{X}$ reveals that C-AlexNet outperforms C-VGG, C-GoogleNet and C-ResNet. This can be explained as:

a. Both C-VGG and C-AlexNet have the same internal layer construction up to the activated $l=2$ cluster but with different parameters i.e. convolutional filter and stride sizes. In fact, C-VGG has two $3 \times 3$ convolutional filters with stride one while C-AlexNet a 11x11 filter size with stride four and a 5x5 with stride two. By comparing the performance on the10-class SOC-1 and 3-class trials, we conclude that the filter size of CVGG does not capture the intra-class spatial content of the target scenes as it is quite small. Even though the $3 \times 3$ convolutional kernel size is sufficient for RGB imagery because it has high-level features (and where VGG is trained for), our trials show that the SAR type data and the capability for intra-class ATR as in the 3-class ATR problem requires larger receptive filters to provide discriminative responses.
TABLE X

3-CLASS ATR WITH VARIOUS CCNN VARIANTS (\%)

\begin{tabular}{lccccc}
\hline \multicolumn{1}{c}{ Method } & BMP2 & BTR70 & T72 & $\begin{array}{c}\text { CCNN } \\
\text { only } \\
\text { (avg.) }\end{array}$ & $l_{1-2}$-CCNN \\
\hline C-VGG & 22.83 & 10.71 & 95.38 & 52.19 & 94.32 \\
C-GoogleNet & 0.33 & 44.93 & 95.72 & 47.51 & 89.21 \\
C-AlexNet & $\mathbf{9 8 . 3 0}$ & $\mathbf{9 8 . 4 8}$ & $\mathbf{9 8 . 6 4}$ & $\mathbf{9 8 . 4 7}$ & $\mathbf{9 9 . 3 3}$ \\
C-ResNet & 10.23 & 81.12 & 92.29 & 61.21 & 91.71 \\
\hline \hline
\end{tabular}

GoogleNet mainly uses inception modules rather than a standard deep network construction. The tuning process of $\mathrm{C}$ GoogleNet provided as optimum cluster the $1=2$ ending with the Pool_2 layer and thus, a quite shallow part of the original GoogleNet is exploited even before the inception modules are applied. For the activated layer $1=2$, the two convolutional filters involve a 7x7 and a 3x3 kernel size and thus similarly to the C-VGG these are too small to encapsulate the intra-class SAR imagery information and bridge the original training with the testing modality gap i.e. visual vs. SAR imagery.

b. ResNet uses residual blocks. Each residual block encloses a convolutional filter of $3 \times 3$, which similarly to the C-VGG and C-GoogleNet, does not encapsulate efficiently the intra-class target variations.

By extending our architecture to facilitate the mainstream CNNs, we can draw the following conclusions. First, our concept is validated since in the SOC-1 ATR problem all CNNs have a similar performance. Second, the size of the convolutional filter plays an important role in the intra-class ATR performance. This is obvious from the 3-class scenario, 
which highlights that the CNNs with a small filter size fail to classify correctly the target.

\section{CONCLUSION}

Deep learning techniques for ATR of SAR imagery aim at extracting deep features that can uniquely describe a target within a SAR image. Instead of a single-discipline solution, we fuse a Convolutional Neural Network module with a Sparse Coding module. For the former, we extend the effectiveness of the AlexNet CNN to operate from the visual to the SAR domain by introducing a layer-clustering concept. In order to bridge the visual-SAR modality gap, the clusteredCNN is combined with a multi-class SVM classification scheme. The latter module (Sparse Coding), extends Sparse Coding theory to facilitate a proposed adaptive elastic net optimization concept that balances the advantages of $l_{1}-$ norm and $l_{2}-$ norm optimization based on the scene SAR imagery. Finally, the Clustered CNN and the adaptive Sparse Coding module are innovatively fused under a decision level scheme that adaptively alters the fusion weights based on the scene characteristics.

Experimental results on the MSTAR data set under various configurations such as the 10-class ATR problem with and without target variants, the 3-class ATR problem, and affected by several nuisance factors such as noise, large depression angle variation and resolution variation, illustrate the effectiveness of our suggested architecture against current ATR techniques. In fact, on the MSTAR dataset, our architecture yields the highest ATR performance reported yet in the literature, which is $99.33 \%$ and $99.86 \%$ for the 3 and 10-class problems respectively. Finally, we also demonstrate that among current CNNs used by the computer vision community, AlexNet has the unique characteristics to host this data modality extension.

\section{REFERENCES}

[1] Gray, G. J., Aouf, N., Richardson, M. A., Butters, B. and Walmsley, R., "An intelligent tracking algorithm for an imaging infrared antiship missile," in Proc. SPIE 8543, Technologies for Optical Countermeasures IX, 2012, vol. 8543, p. 85430L-85430L.

[2] Kechagias-Stamatis, O., Aouf, N., and Richardson, M. A., "3D automatic target recognition for future LIDAR missiles," IEEE Trans. Aerosp. Electron. Syst., vol. 52, no. 6, pp. 2662-2675, Dec. 2016

[3] Kechagias-Stamatis, O., Aouf, N., "Evaluating 3D local descriptors for future LIDAR missiles with automatic target recognition capabilities," Imaging Sci. J., vol. 65, no. 7, pp. 428-437, Aug. 2017.

[4] Kechagias-Stamatis, O., Aouf, N., Gray, G., Chermak, L., Richardson, M. A, and Oudyi, F., "Local feature based automatic target recognition for future 3D active homing seeker missiles," Aerosp. Sci. Technol., vol. 73, pp. 309-317, Feb. 2018

[5] Chen, S., Wang, H., Xu, F., and Jin, Y.-Q., "Target Classification Using the Deep Convolutional Networks for SAR Images," IEEE Trans. Geosci. Remote Sens., vol. 54, no. 8, pp. 4806-4817, Aug. 2016.

[6] Zhong, Y. and Ettinger, G., "Enlightening Deep Neural Networks with Knowledge of Confounding Factors," arXiv Prepr. arXiv1607.02397, pp. 1-10, 2016

[7] Wilmanski, M., Kreucher, C. and Lauer, J., "Modern approaches in deep learning for SAR ATR," in Proc. SPIE, 2016, vol. 9843, p. $98430 \mathrm{~N}$.
Chen S. and Wang, H., "SAR target recognition based on deep learning," in 2014 International Conference on Data Science and Advanced Analytics (DSAA), 2014, pp. 541-547.

Paladini, R., Martorella, M. and Berizzi, F., "Classification of ManMade Targets via Invariant Coherency-Matrix Eigenvector Decomposition of Polarimetric SAR/ISAR Images," IEEE Trans. Geosci. Remote Sens., vol. 49, no. 8, pp. 3022-3034, Aug. 2011.

Perissin, D. and Ferretti, A., "Urban-Target Recognition by Means of Repeated Spaceborne SAR Images," IEEE Trans. Geosci. Remote Sens., vol. 45, no. 12, pp. 4043-4058, Dec. 2007.

Dong, G., Wang, N. and Kuang, G., "Sparse Representation of Monogenic Signal: With Application to Target Recognition in SAR Images," IEEE Signal Process. Lett., vol. 21, no. 8, pp. 952-956, Aug. 2014.

Profeta, A., Rodriguez, A. and Clouse, H. S., "Convolutional neural networks for synthetic aperture radar classification," in In SPIE Defense + Security International Society for Optics and Photonics, 2016, vol. 9843 , p. $98430 \mathrm{M}$.

Morgan, D. A. E. "Deep convolutional neural networks for ATR from SAR imagery," SPIE Def. + Secur., vol. 9475, p. 94750F, 2015.

Zhang, X., Qin, J. and Li, G., "SAR target classification using Bayesian compressive sensing with scattering centers features," Prog. Electromagn. Res., vol. 136, pp. 385-407, 2013.

Doo, S., Smith, G., Baker, C., Cui, Z., Feng, J., Cao, Z., "Aspect invariant features for radar target recognition," IET Radar, Sonar Navig., vol. 11, no. 4, pp. 597-604, Apr. 2017.

Darymli, K., McGuire, P., W. Gill, E., Power, D. and Moloney, C., "Holism-based features for target classification in focused and complex-valued synthetic aperture radar imagery," IEEE Trans. Aerosp. Electron. Syst., vol. 52, no. 2, pp. 786-808, 2016.

Deng, S., Du, L., Li, C., Ding, J. and Liu, H., "SAR Automatic Target Recognition Based on Euclidean Distance Restricted Autoencoder," IEEE J. Sel. Top. Appl. Earth Obs. Remote Sens., vol. 10, no. 7, pp. 3323-3333, Jul. 2017.

Kang, M., Ji, K., Leng, X., Xing, X. and Zou, H., "Synthetic Aperture Radar Target Recognition with Feature Fusion Based on a Stacked Autoencoder," Sensors, vol. 17, no. 12, p. 192, Jan. 2017.

Cui, Z., Feng, J., Cao, Z., Ren, H. and Yang, J., "Target recognition in synthetic aperture radar images via non-negative matrix factorisation," IET Radar, Sonar Navig., vol. 9, no. 9, pp. 13761385, Dec. 2015.

Clemente, C., Pallotta, L., Gaglione, D., De Maio, A. and Soraghan, J. J., "Automatic Target Recognition of Military Vehicles With Krawtchouk Moments," IEEE Trans. Aerosp. Electron. Syst., vol. 53, no. 1, pp. 493-500, Feb. 2017.

Huang,, X., Nie, X., Wu, W., Qiao, H. and Zhang, B., "SAR target configuration recognition based on the biologically inspired model," Neurocomputing, vol. 234, pp. 185-191, Apr. 2017.

Yang, S., Wang, M., Long, H. and Liu, Z., "Sparse Robust Filters for scene classification of Synthetic Aperture Radar (SAR) images," Neurocomputing, vol. 184, pp. 91-98, Apr. 2016.

Ding, B., Wen, G., Ma, C. and Yang, X., "Target recognition in synthetic aperture radar images using binary morphological operations," J. Appl. Remote Sens., vol. 10, no. 4, p. 046006, Oct. 2016.

Ding, B., Wen, G., Zhong, J., Ma, C. and Yang, X., "A robust similarity measure for attributed scattering center sets with application to SAR ATR," Neurocomputing, vol. 219, pp. 130-143, Jan. 2017.

Gorovyi, I. M. and Sharapov, D. S., "Efficient object classification and recognition in SAR imagery," in 2017 18th International Radar Symposium (IRS), 2017, pp. 1-7.

$\mathrm{Ni}$, J. C. and Xu, Y. L., "SAR automatic target recognition based on a visual cortical system," in 2013 6th International Congress on Image and Signal Processing (CISP), 2013, vol. 2, pp. 778-782.

Sun,, Z., Xue, L. and Xu, Y., "Recognition of SAR target based on multilayer auto-encoder and SNN," Int. J. Innov. Comput. Inf. Control, vol. 9, no. 11, pp. 4331-4341, 2013.

Liu, S., Zhan, R., Zhai, Q., Wang, W. and Zhang, J., "Multi-view radar target recognition based on multitask compressive sensing," J. Electromagn. Waves Appl., vol. 29, no. 14, pp. 1917-1934, 2015.

Zhang, H., Nasrabadi, N. M., Zhang, Y. and Huang, T. S., "Multiview automatic target recognition using joint sparse representation," IEEE Trans. Aerosp. Electron. Syst., vol. 48, no. 3, pp. 2481-2497, 
2012.

[30] Wang, H., Chen, S., Xu, F. and Jin, Y.-Q., "Application of deeplearning algorithms to MSTAR data," in 2015 IEEE International Geoscience and Remote Sensing Symposium (IGARSS), 2015, pp. 3743-3745.

[31] Yonel, B., Mason, E. and Yazici, B., "Deep Learning for Passive Synthetic Aperture Radar," IEEE J. Sel. Top. Signal Process., vol. 12, no. 1, pp. 90-103, Feb. 2018.

[32] Krizhevsky, A., Sutskever, I. and Hinton, G., "Imagenet classification with deep convolutional neural networks," in Advances in neural information processing systems, 2012, pp. 1097-1105.

[33] Simonyan, K. and Zisserman, A., "Very Deep Convolutional Networks for Large-Scale Image Recognition," arXiv Prepr. arXiv1409.1556v6, pp. 1-14, Sep. 2014.

[34] Szegedy, C., Wei L., Yangqing J., Sermanet, P., Reed, S., Anguelov, D., Erhan, D., Vanhoucke, V., Rabinovich, A., "Going deeper with convolutions," in 2015 IEEE Conference on Computer Vision and Pattern Recognition (CVPR), 2015, pp. 1-9.

[35] He, K., Zhang, X., Ren, S. and Sun, J., "Deep Residual Learning for Image Recognition," in 2016 IEEE Conference on Computer Vision and Pattern Recognition (CVPR), 2016, pp. 770-778.

[36] Baraniuk, R., "Compressive Sensing [Lecture Notes]," IEEE Signal Process. Mag., vol. 24, no. 4, pp. 118-121, Jul. 2007.

[37] Dong, G. and Kuang, G., "Classification on the Monogenic Scale Space: Application to Target Recognition in SAR Image," IEEE Trans. Image Process., vol. 24, no. 8, pp. 2527-2539, Aug. 2015.

[38] Candes, E. J., Romberg, J. and Tao, T., "Robust uncertainty principles: exact signal reconstruction from highly incomplete frequency information," IEEE Trans. Inf. Theory, vol. 52, no. 2, pp. 489-509, Feb. 2006

[39] Wright, J., Yang, A. Y., Ganesh, A., Sastry, S. S. and Ma, Y., "Robust Face Recognition via Sparse Representation," IEEE Trans. Pattern Anal. Mach. Intell., vol. 31, no. 2, pp. 210-227, Feb. 2009.

[40] Barazandeh, B., Bastani, K., Rafieisakhaei, M., Kim, S., Kong, Z. and Nussbaum, M. A., "Robust Sparse Representation-Based Classification Using Online Sensor Data for Monitoring Manual Material Handling Tasks," IEEE Trans. Autom. Sci. Eng., pp. 1-12, 2017.

[41] McLachlan, G. and Peel, D., Finite mixture models. New York, USA: John Wiley \& Sons, 2004

[42] Zou, H. and Hastie, T., "Regularization and variable selection via the elastic net," J. R. Stat. Soc. Ser. B (Statistical Methodol., vol. 67, no. 2, pp. 301-320, Apr. 2005.

[43] Friedman, J., Hastie, T. and Tibshirani, R., "Regularization Paths for Generalized Linear Models via Coordinate Descent," J. Stat. Softw., vol. 33, no. 1, 2010.

[44] Pan, S. J. and Yang, Q., “A Survey on Transfer Learning," IEEE Trans. Knowl. Data Eng., vol. 22, no. 10, pp. 1345-1359, Oct. 2010.

[45] Kang, C. and He, C., "SAR image classification based on the multilayer network and transfer learning of mid-level representations," in 2016 IEEE International Geoscience and Remote Sensing Symposium (IGARSS), 2016, pp. 1146-1149.

[46] Marmanis, D., Datcu, M., Esch, T. and Stilla, U., "Deep Learning Earth Observation Classification Using ImageNet Pretrained Networks," IEEE Geosci. Remote Sens. Lett., vol. 13, no. 1, pp. 105-109, Jan. 2016.

[47] Zha, S., Luisier, F., Andrews, W., Srivastava, N. and Salakhutdinov, R., "Exploiting Image-trained CNN Architectures for Unconstrained Video Classification," in Procedings of the British Machine Vision Conference 2015, 2015, p. 60.1-60.13.

[48] Schwarz, M., Schulz, H. and Behnke, S., "RGB-D object recognition and pose estimation based on pre-trained convolutional neural network features," in 2015 IEEE International Conference on Robotics and Automation (ICRA), 2015, no. May, pp. 1329-1335.

[49] Mayoraz, E. and Alpaydin, E., "Support vector machines for multiclass classification," in Engineering Applications of Bio-Inspired Artificial Neural Networks, 1999, pp. 833-842.

[50] "ImageNet." [Online]. Available: http://www.image-net.org/. [Accessed: 15-Aug-2015].

[51] Wu, J., "Introduction to Convolutional Neural Networks," Natl. Key Lab Nov. Softw. Technol., pp. 1-28, Nov. 2016.

[52] Lindeberg, T., "Scale-space theory: a basic tool for analyzing structures at different scales," J. Appl. Stat., vol. 21, no. 1, pp. 225270, 1994.
Glorot, X., Bordes, A. and Bengio, Y., "Deep Sparse Rectifier Neural Networks," in Proceedings of the Fourteenth International Conference on Artificial Intelligence and Statistics, 2011, vol. 15, pp. 315-323.

[54] Wang, Z. and Xue, X., "Multi-Class Support Vector Machine," in Support Vector Machines Applications, Cham: Springer International Publishing, 2014, pp. 23-48.

[55] Ross, T. D., Worrell, S. W., Velten, V. J., Mossing, J. C. and Bryant, M. L., "Standard SAR ATR Evaluation Experiments using the MSTAR Public Release Data Set," in SPIE Conference on Algorithms for Synthetic Aperture Radar Imagery V, 1998, vol. 3370, pp. 566-573.

[56] Vedaldi, A. and Lenc, K., "MatConvNet," in Proceedings of the 23rd ACM international conference on Multimedia - MM '15, 2015, pp. 689-692.

[57] Bolourchi, P., Demirel, H. and Uysal, S., "Target recognition in SAR images using radial Chebyshev moments," Signal, Image Video Process., vol. 11, no. 6, pp. 1033-1040, Sep. 2017.

[58] Liu, H. and Li, S., "Decision fusion of sparse representation and support vector machine for SAR image target recognition," Neurocomputing, vol. 113, pp. 97-104, Aug. 2013.

[59] Zhang, R., Hong, J., Ming, F., Zhang, R., Hong, J. and Ming, F., "An improved PCA based features for SAR ATR," in IET Conference Publications, 2009, no. 551 CP, pp. 313-313.

[60] Ding, B., Wen, G., Ye, F., Huang, X. and Yang, X., "Feature extraction based on 2D compressive sensing for SAR automatic target recognition," in 2017 11th European Conference on Antennas and Propagation (EUCAP), 2017, pp. 1219-1223.

[61] Srinivas, U., Monga, V. and Raj, R. G., "SAR Automatic Target Recognition Using Discriminative Graphical Models," IEEE Trans. Aerosp. Electron. Syst., vol. 50, no. 1, pp. 591-606, Jan. 2014.

[62] Anagnostopoulos, G. C., "SVM-based target recognition from synthetic aperture radar images using target region outline descriptors," Nonlinear Anal. Theory, Methods Appl., vol. 71, no. 12, pp. 2934-2939, Dec. 2009.

[63] Rezai-rad, G. and Amoon, M., "Automatic target recognition of synthetic aperture radar (SAR) images based on optimal selection of Zernike moments features," IET Comput. Vis., vol. 8, no. 2, pp. 7785, Apr. 2014.

[64] Wagner, S. A., "SAR ATR by a combination of convolutional neural network and support vector machines," IEEE Trans. Aerosp. Electron. Syst., vol. 52, no. 6, pp. 2861-2872, Dec. 2016.

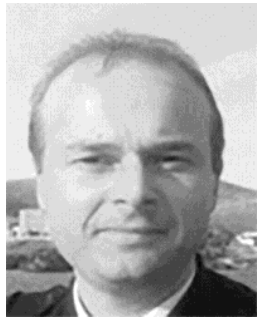

Odysseas Kechagias-Stamatis received the MSc degree in Guided Weapon Systems and the $\mathrm{PhD}$ degree in 3D ATR for missile platforms from Cranfield University, U.K. in 2011 and 2017 respectively. His research interests include 2D/3D object recognition and tracking, data fusion and autonomy of systems.

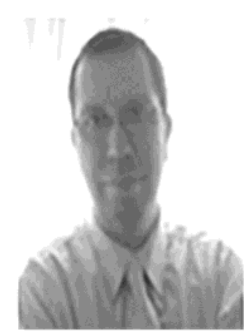

Professor Nabil Aouf is currently Head of the Signals and Autonomy group, Centre for Electronic Warfare information and Cyber, Cranfield University, U.K. He has authored over 100 publications in high caliber in his domains of interest. His research interests are aerospace and defense systems, information fusion and vision systems, guidance and navigation, tracking, and control and autonomy of systems. He is an Associate Editor of the International Journal of Computational Intelligence in Control. 